\title{
Mitochondrial dynamics in the regulation of neuronal cell death
}

\author{
Eric C. C. Cheung $\cdot$ Heidi M. McBride $\cdot$ Ruth S. Slack
}

Published online: 15 February 2007

(C) Springer Science + Business Media, LLC 2007

\begin{abstract}
Mitochondria undergo continuous fission and fusion events in physiological situations. Fragmentation of mitochondria during cell death has been shown to play a key role in cell death progression, including release of the mitochondrial apoptotic proteins. Ultrastructural changes in mitochondria, such as cristae remodeling, is also involved in cell death initiation. Here, we emphasize the important role of mitochondrial fission/fusion machinery in neuronal cell death. Unlike many other cell types such as immortalized cell lines, neurons are distinct morphologically and functionally. We will discuss how this uniqueness presents special challenges in the cellular response to neurotoxic stresses, and how this affects the mitochondrial dynamics in the regulation of cell death in neurons.
\end{abstract}

Keywords Apoptosis · Mitochondria · Mitochondrial fission/fusion · Neurons · Excitotoxicity · Oxidative stress · DNA damage

\section{Introduction}

Mitochondria serve as an important organelle for the control of metabolism, calcium signaling and cell death. It has

E. C. C. Cheung · R. S. Slack $(\bowtie)$

University of Ottawa, Department of Cellular Molecular

Medicine, Ottawa Health Research Institute,

451 Smyth Road, Ottawa, Ontario, K1H 8M5

e-mail: ruth.slack@uottawa.ca

H. M. McBride

University of Ottawa Heart Institute,

Canada been well documented that cell death signals converge on the mitochondria to execute and amplify the message for the cell's demise. The key players in the cell death pathway are the Bcl-2 family members, in which the balance between the antiapoptotic members and the apoptotic members influence the ultimate fate of the cell. Once the balance is shifted towards apoptosis, the mitochondria release apoptotic proteins such as cyt- $c$, EndoG and AIF (for review see [1,2]). In physiological situations, mitochondria are constantly dividing and fusing, which is important in cell division, metabolism, and development. While the exact mechanism of fission and fusion is not clear yet, recent progress has suggested that fission plays an important role in the regulation of cell death. Unlike many other cell types, neurons are distinct since they have long and dynamic processes extending far from the cell body. These processes have a complex repertoire of ion receptors and channels which respond to external signals such as neurotransmitters. During neurotoxic insults such as excitotoxicity and ischemia/reperfusion, a pathologically high level of neurotransmitters such as glutamate activates these receptors and cell death is initiated by distinct mechanisms which do not always depend on the classical Bcl-2 apoptosis machinery. Neurons, therefore, have unique challenges in the cellular response to cytotoxic stress such as those induced after stroke or traumatic injury. In the following review we will provide an overview of neuronal cell death, the fission and fusion mechanism in mammalian systems, and finally a discussion regarding the potential mechanisms by which the mitochondrial fission and fusion machinery can modulate neuronal cell death. Understanding the mechanisms by which these proteins contribute to neuronal cell death will provide new insights into neuroprotective strategies applicable to the treatment of acute brain injury and neurodegeneration. 


\section{Overview of neuronal cell death}

There are two major mechanisms contributing to cell death in neurons following acute brain injury: (a) the classical apoptotic cell death which involves caspases, and (b) excitotoxic cell death [3-5]. Both of these pathways converge on the mitochondria: the classical apoptotic cell death involving release of cyt $c$ from mitochondria that leads to caspase activation; and, excitotoxic cell death which induces $\mathrm{Ca}^{2+}$ influx, causing mitochondrial dysfunction and the production of reactive oxygen species. Excitotoxic neuronal injury recruits both apoptotic and necrotic cell death machineries that can be inhibited by inactivation of proapoptotic molecules $[6,7]$ (for review see $[4,5]$ ). Both of these pathways are key contributors to neuronal cell death during acute brain injury such as stroke.

Classical apoptotic cell death involves members of the Bcl-2 family proteins. The multi domain Bcl-2 proapoptotic proteins $\mathrm{Bax}$ and $\mathrm{Bak}$ are activated by $\mathrm{BH} 3$ only proteins which lead to the induction of mitochondrial outer membrane permeabilization (MOMP) [1, 2]. Once MOMP occurs, pro-apoptotic factors from the intermembrane space such as cytochrome $C$ and Smac/Diablo are released. Cytochrome $C$, with Apaf1 and pro-caspase 9, activates the caspase cascade which leads to caspase dependent apoptosis. Other caspase independent factors, such as AIF, are also released from the mitochondrial intermembrane space, which is the major death executioner in caspase independent pathways or when caspases are inhibited [3]. One of the major triggers of mitochondrial Bax activation following neuronal injury is DNA damage. In neuronal cell death, reactive oxygen species can be generated by multiple mechanisms such as hypoxia that causes a lack of oxygen to produce ATP. This condition, common in stroke, leads to mitochondrial dysfunction and the subsequent production of ROS when oxygen level is high in the reoxygenation stage. Exposure to elevated ROS causes DNA damage which can in turn trigger apoptotic signalling such as p53 activation that signals through Bcl-2 family proteins. Proapoptotic Bcl family proteins converge on the mitochondria to induce cytochrome $c$ release which triggers the caspase cascade leading to the orderly breakdown of the cell.

In neurons, acute brain injury after stroke and traumatic brain injury also leads to excitotoxic cell death which exhibits partial apoptotic-necrotic like features and does not require $\mathrm{Bax} / \mathrm{Bak}$ to initiate cell death [8]. While excitotoxic cell death also converges on mitochondria, the upstream signalling events are quite distinct. We and others have shown that this mode of cell death occurs independent of Bax/Bak because deletion of these proteins does not affect the rate of cell death [8-10]. During acute brain injury such as stroke, oxygen levels are reduced, which leads to an abrupt decrease in ATP production and rapid depolarization of the plasma membrane which occurs early after the ischemic insult in stroke. The level of glutamate in the synapses is controlled by channels that rely heavily on energy produced by ATP, therefore, a reduction of ATP during stroke causes the release of abnormally high amounts of glutamate into the synapses [11]. Excessive gluamate in the synapses activates the ionotrophic glutamate receptors to a pathophysiological level and this type of neuronal insult is called excitotoxicity $[12,13]$. Activation of glutamate receptors induces influx of $\mathrm{Na}^{+}$ion, which causes further depolarization, and release of glutamate. This, coupled with impaired reuptake of glutamate due to lack of ATP, exacerbates the excitotoxic response through sustained activation of glutamate receptors [14-18]. While there are several receptors for excitatory amino acids, NMDA receptors are believed to be the key mediators of death during excitotoxic injury whereby glutamate overstimulation leads to excessive $\mathrm{Ca}^{2+}$ influx and elevation of cytoplasmic $\mathrm{Ca}^{2+}$ levels (reviewed in [19]). $\mathrm{Ca}^{2+}$ influx into the cytoplasm has been shown to activate second messengers, one of which is neuronal nitric oxide synthase (nNOS) [2022]. Activation of nNOS leads to the generation of excessive levels of nitric oxide (NO). NO serves as a substrate for the production of highly reactive free radicals such as peroxynitrites which promote cellular damage and ultimately neuronal cell death [23-26]. Excess $\mathrm{Ca}^{2+}$ also enters the mitochondria and induces mitochondrial dysfunction which leads to the release of the pro-apoptotic intermembrane proteins such as cyt $c$ [27]. All of these mechanisms exacerbate the levels of ROS during excitotoxicity and ultimately leads to cell death in a necrotic/apoptotic fashion, which is distinct from the classical apoptotic cell death that mainly involves the Bcl-2 family protein such as Bax and Bak. During acute neuronal injury such as stroke, neurons thus face distinct challenges involving diverse mechanisms of cell death which trigger classical Bax/Bak dependent apoptosis as well as Bax/Bak-independent pathways. Recent studies suggest that the mitochondrial fission and fusion machinery plays a crucial role in the regulation of multiple mechanisms of neuronal cell death that occur following injury, which will be the focus of this review.

\section{Overview of mitochondrial membrane dynamics machine}

Mitochondria are dynamic organelles that constantly undergo fission and fusion events and are transported to strategic locations within the cell. Recent studies suggest that mitochondrial dynamics are closely coupled to the regulation of cell death [28-33]. It is, however, still unclear whether mitochondrial fission is sufficient and/or necessary for cell death to occur; or what the exact role of mitochondrial fission may be in regulating mitochondrial permeabilization 
Table 1 Mitochondrial dynamic proteins that are discussed in this review for details see text

\begin{tabular}{llll}
\hline Mammals & Yeast & Role in mitochondrial dynamics & Location \\
\hline Mfn1/2 & Fzo1p & Fusion & Outer mitochondrial membrane \\
Opa1 & Mgm1p & Fusion & Inner mitochondrial membrane facing intermembrane space \\
Paraplegin & $?$ & Fusion Opa1 processor & Inner membrane protein \\
PARL & Rbd1p/Pcp1p/Mdm38p & Fusion Opa1 processor & Inner membrane protein \\
Drp1 & Dnm1p & Fission & Mostly cytosolic, then recruited to outer membrane in puncta \\
& & & during fission \\
Fis1 & Fis1p & Fission & Outer mitochondrial membrane \\
Endophilin B1 & $?$ & Fission & Mostly cytosolic, then recruited to mitochondria during fission \\
$?$ & Mdv1p & Fission & Outer mitochondrial membrane \\
$?$ & Caf4p & Fission & Outer mitochondrial membrane \\
\hline
\end{tabular}

during cell death. In the following section we will review the fission/fusion machinery focusing on the proteins that have been shown to be involved in mammalian cell death (Table 1). For a more detailed discussion of mitochondrial fission/fusion machinery see review [34-40].

\section{Mitochondrial fission and cell death}

Mitochondrial fission is an ongoing process in healthy cells and is essential for cell division, calcium regulation and development [32, 41-43]. In neurons, mitochondrial fission is essential for mitochondrial transport to their potential docking sites in axons and dendrites [44, 45]. Immature neurons have smaller, more motile mitochondria whereas mature neurons with established synaptic connections exhibited longer, and more stationary mitochondria, strategically placed in regions of high energy demand [44]. In addition to the physiological role of mitochondrial fission, recently fission has also been implicated in cell death pathway $[46,47]$. Here we will first review the mammalian fission proteins that have been implicated in the cell death pathway, and we will discuss how these fission proteins regulate neuronal cell death.

\section{Mitochondrial fission machinery in mammalian system}

While many molecules comprising the fission machinery have been identified in yeast, only three mammalian orthologues have been found so far. Fis1, Drp1, and Endophilin B1 are known to be required for mitochondrial fission in mammals (Table 1). Other mammalian proteins such as DAP3, GDAP1 and MTP18 may also participate in mitochondrial fission, however, their yeast homologues have not been found and their exact roles in fission are still not clear [48-50]. Fis 1 is distributed evenly on the outer mitochondrial membrane. In physiological situation, it functions with Drp1 and contains a cytosolic domain believed to interact with proteins that trigger fission $[51,52]$. Drp1 is a cytosolic dynamin re- lated GTPase that is recruited to the mitochondrial surface where it is distributed in punctate foci along the tubules, many of which localize to sites of fission [53, 54]. The oligomerized Dnm1p (yeast ortholog of Drp1) can form spirals that are the size of mitochondrial fission sites and can constrict artificial liposomes to a diameter that fits around the $100 \mathrm{~nm}$ mitochondria [53]. Endophilin B1, a cytosolic protein, is also required for inner membrane fission and membrane remodeling during fission [55].

Yeast mitochondria, unlike the mammalian system, utilized additional adaptor protein such as Caf4p and Mdv1p for mitochondrial fission [54]. Orthologues of these proteins in mammalian system have not been found. In mammalian cells, a weak interaction between Fis1 and Drp1 has been detected using cross linking and overexpressed proteins [52, 56]. While these studies suggest that Fis1 helps to facilitate Drp1 recruitment, it has also been shown that mitochondrial recruitment of Drp1 can still occur in the absence of Fis1 [57]. Based on the finding that the interaction between Fis 1p and Dnm1p in yeast is indirect, it is most likely that mammalian systems have evolved similar adapter proteins to facilitate the interaction between Fis1 and Drp1. It has also been shown that Drp1 is post-translationally modified by SUMOylation, which plays a role in the regulation of mitochondrial fission [58]. Presently in mammalian cells, the mechanisms by which (i) cytosolic Drp1 becomes activated and recruited to the mitochondria to induce fission; and (ii) the proteins complexes interacting with Drp1 and Fis1 to execute fission, remain unclear.

\section{What is the role of mitochondrial fission in cell death?}

It is well documented that fission accompanies most modes of cell death. Earlier studies involving electron microscopy revealed that mitochondria become fragmented after induction of cell death by various stimuli [59-61]. As cell imaging techniques became more advanced, time lapse photography revealed that mitochondrial fission coincided very closely 
with the induction of cell death. Fission was observed around the time of Bax activation at the mitochondria, but before the mitochondrial outer membrane permeabilization (MOMP) and the release of cytochrome $C$ [62]. These studies, arising from multiple apoptotic systems, suggest a causal link between mitochondrial fission and the execution of cell death.

The implication that fission may be a requirement for the apoptotic process came from studies examining the role of Drp1 [28, 30, 31, 33, 57, 63]. Drp1 was found to be colocalized with Bax at the sites of future fission after cell death induction. Moreover, inhibition of Drp1 activity by expression of the dominant negative mutant could prevent mitochondrial fission, cytochrome $c$ release, and the subsequent cell death [28]. Another study examining an ER initiated apoptotic pathway revealed that inhibition of Drp1 activity could also prevent mitochondrial fission and cell death $[63,64]$. More recently, downregulation of the mitochondrial membrane bound fission protein, Fis1, could prevent mitochondrial fission and cell death [57]. Another protein involved in fission, Endophilin B1, was shown to translocate to the mitochondria during cell death and is required for the activation of $\mathrm{Bax}$ on the mitochondria, likely due to their direct interactions on the membrane during cell death [55, 65]. Finally, knockdown of DAP3, a matrix protein shown to induce mitochondrial fission during cell death could inhibit fission and protect cells against apoptosis [48]. Taken together, these studies provide extensive evidence in support of a role for mitochondrial fission proteins such as Drp1 and Fis1 in the regulation of cell death.

Although compelling evidence supports a key role for mitochondrial fission in the regulation of cell death, one might ask whether MOMP, cytochrome $C$ release and cell death can proceed in the absence of fission. In some cell death scenarios, fission is not a requirement for cytochrome $C$ release. For example in Bax treated protozoan Trypanosoma brucei, and HeLa cells treated with actinomycin D which evokes Bax/Bak mediated cell death [66], cytochrome $C$ release precedes mitochondrial fragmentation [67, 68]. In another study, Bcl-2 has been shown to inhibit cell death but not mitochondrial fission [69]. The fact that Bax alone can induce release of molecules from liposomes, suggests that Bax does not require mitochondria to undergo fission to form pores for the release of proapoptotic proteins [70]. Recent studies in C. elegans [71] have clearly disconnected the fission event from apoptosis where they have shown that cells with completely fragmented mitochondria can survive. Inhibition of fragmentation by downregulation of Drp1 or Fis1 does not inhibit Bax/Bak dependent apoptosis and can only partially inhibit release of cytochrome $C$ [72]. One explanation is that molecules that play pivotal roles in the regulation of fission may have dual functions and may participate in cell death signalling independent of their roles in mitochondrial fission. This is exemplified by a recent study by the Scor- rano group which separated the apoptosis inducing domain and fission inducing domain of Fis1. In this work, it was demonstrated that expression of fission dead Fis 1 could still induce cell death. When the apoptotic dead mutant of Fis1 was expressed, mitochondria could still undergo fission but there is no induction of cell death [73]. Overall, these results show that although fission is required for cell death, fission in itself was not sufficient for cell death induction. One may envisage, therefore, a model in which the fission machinery is required for apoptosis and is recruited during apoptosis signalling to induce cell death by as of yet unidentified mechanisms. One possibility is that mitochondrial fission may participate in cell death by modifying the lipid microenvironment or deform lipid bilayers for Bax/Bak induced mitochondrial permeabilization $[74,75]$. While the pro-apoptotic role for mitochondrial fragmentation remains unclear, all of these studies support an important role of fission proteins in the execution of cell death. This duality of function among fission proteins appears to be a recurring theme in mitochondrial dynamics, and will re-emerge when the role of proteins required for mitochondrial fusion are discussed below.

Different scenarios have been proposed as to how mitochondrial fission might participate in the execution of cell death (Fig. 1). It has been suggested that fission proteins may interact with Bax during apoptosis. Drp1, Mfn2, and Bax colocalize at fission sites during cell death [33]. Drp1 translocation to mitochondria, however, is not dependent on Bax [69], and Bax recruitment is not inhibited by inactivation of Drp1 [28, 33, 57]. In addition, no direct interaction has been observed between Bax and Drp1. Inhibition of Fis1, however, has been shown to disrupt Bax translocation and its activation at the mitochondria, which places these fusion proteins upstream of Drp1, at least within the apoptotic program [57]. It is possible that Endophilin B1, a Bax interactor during apoptosis, participates in the co-localization of Drp1 and Bax at the mitochondria during apoptosis, since Endophilin $\mathrm{B} 1$ is redistributed to mitochondria after cell death induction [55], although Endophilin B1 interacts with Bax mainly in the cytosol and does not significantly influence Bax mediated cell death [65]. Other proapoptotic factors, therefore, may be recruited to the mitochondrial to drive apoptosis facilitated by the fission machinery. An alternative view of how mitochondria fission machinery is activated in apoptosis comes from several studies indicating that the release of some mitochondrial factors such as DDP/TIMM8a can activate mitochondrial fission. In a similar fashion, the mitochondrial fusion protein Opa1 is also released and hence may disrupt the fusion machinery which also leads to increased fission [31, 68]. This scenario, however, is inconsistent with the inability of $\mathrm{Bcl} 2$ to inhibit mitochondrial fission, which blocks cell death from mitochondria [69]. A detailed comparison of the mitochondrial fission complexes during cell 


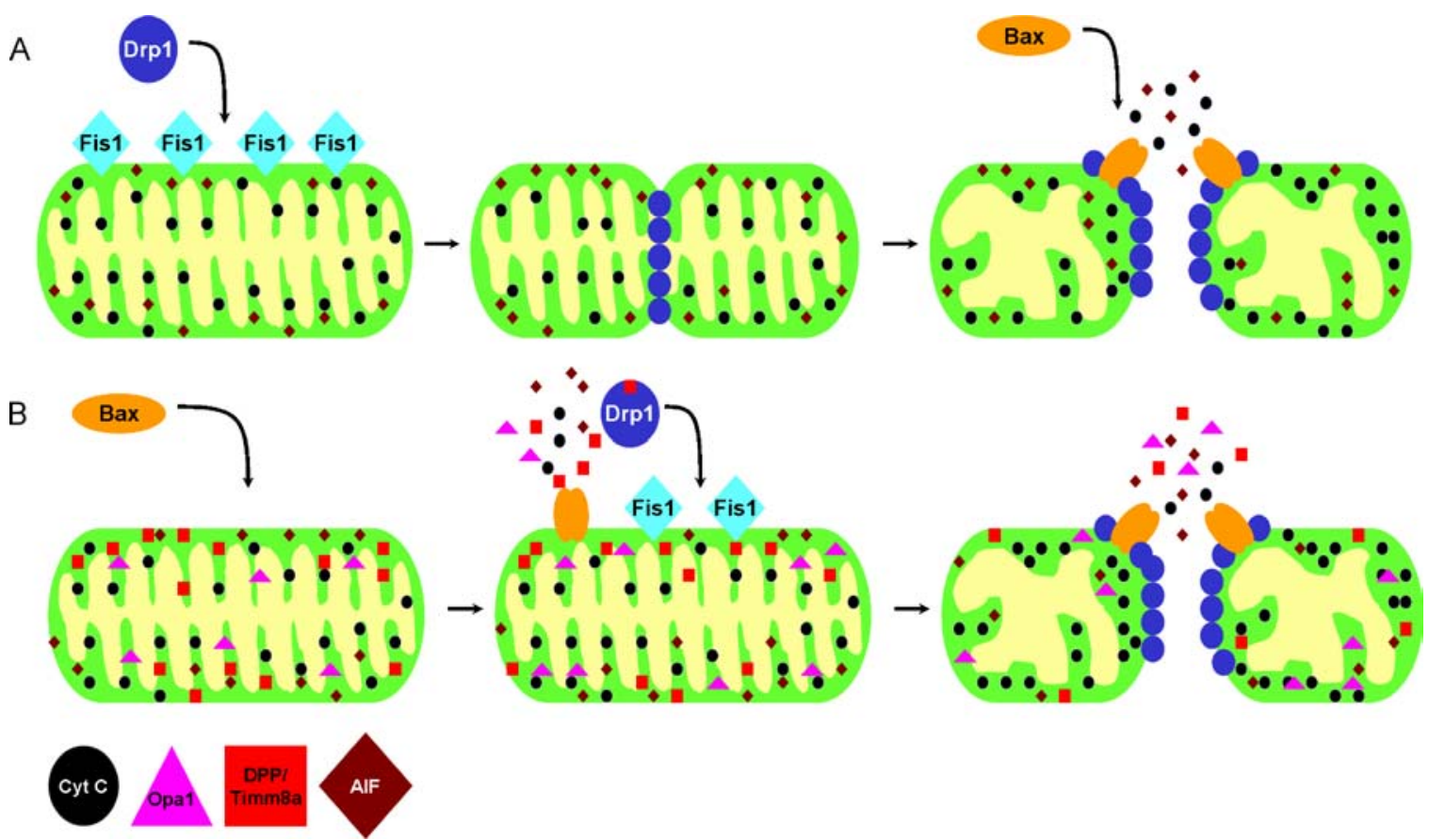

Fig. 1 Two scenarios for the role of mitochondrial fission in apoptosis. (A) Drp1 is recruited to the mitochondria during cell death, then Bax is translocated to the fission site. (B) Bax is first recruited to the mitochon-

death with that during normal physiological fission may shed light on the mechanisms by which the mitochondrial fission machinery becomes activated during cell death. The participation of the fission protein Drp1 and Fis1 is undoubtedly an important event in the execution of cell death.

\section{How does mitochondrial fission contribute to neuronal cell death?}

Recently, Barsoum and colleagues have shown that similar to cell lines, neurons also exhibited fragmented mitochondria following exposure to nitric oxide. Mitochondrial fragmentation was an early event that occurred before release of mitochondrial proteins and neurite degeneration. Similar observations were also made in an in vivo animal model of stroke [30] which includes both caspase dependent and independent mechanisms of cell death. Furthermore, expression of fission proteins such as Fis1 and Drp1 could induce mitochondrial fission and the onset of cell death in postmitotic neurons. Conversely, expression of a dominant negative Drp1 could block mitochondrial fission and enhance the rate of neuronal survival after exposure to reactive oxygen species [30]. Studies by our group have also found that mitochondrial fission in neurons undergoing ROS and DNA damage occurs early in cell death. Expression of an activated species of Mfn2 could enhance fusion and protect neurons against cell death induced by DNA damage as well as ROS [76]. These stud- dria and induce MOMP, which releases the fusion protein Opa1 and disrupt the fusion machinery, and DPP/Timm8a for activating Drp1. For details see the text

ies indicate that inhibition of mitochondrial fission after cell death initiation could protect neurons against various types of acute neuronal injury.

\section{Mitochondrial fusion and cell death}

As with mitochondrial fission, the fusion machinery also plays an essential role in development and survival [35, 77, 78]. Fusion is thought to protect mitochondrial function by allowing rapid mixing of membranes, mitochondrial DNA and soluble contents that may be damaged by localized deficits of substrates as a result of cellular stress [35]. Recently, several studies indicated that during cell death mitochondrial fusion is inhibited and activation of the fusion machinery could slow down the rate of cell death $[29,69$, 79]. There are, however, some very significant differences in the action of the fusion proteins in the regulation of cell death. In this section we will first give a brief overview of the fusion machinery proteins, followed by a discussion regarding how the different fusion proteins may promote cell survival.

\section{Mitochondrial fusion proteins}

The proteins required for fusion in mammalian cells include Mitofusin 1 (Mfn1), Mitofusin 2 (Mfn2), and Opa1 
(Table 1). Mfn1 and Mfn2 are homologous GTPases located on the mitochondrial outer membrane [80-82]. The loss of Mfn1 or Mfn2 results in embryonic lethality in mice with the embryonic fibroblasts exhibiting a fragmented phenotype where many organelles lose their electrochemical potential and eventually mtDNA [77, 78]. Recently it was also shown that the loss of both Mfn1 and Mfn2 by RNAi results in a complete inhibition of mitochondrial respiration, with oxygen consumption levels reduced to nearly zero, and ATP production severely compromised [77]. In addition, mutations in Mfn2 have been shown to result in Charcott-Marie Tooth Type 2 and 4 in human patients, resulting in a specific form of neuromuscular degeneration [83]. These findings underscore the importance of Mfn1 and Mfn2 in the control of mitochondrial fusion which is important in neuronal survival. Although Mfn1 and Mfn2 are homologues, there are some important functional differences. For example, Mfn1, but not Mfn2 genetically interacts with Opa1 for fusion, as well as having a higher GTPase activity than Mfn2 [84, 85]. Mfn2 deficient mice have a defect in the giant trophoblast layer of the placenta that causes the embryonic lethality whereas the Mfn1 deficient mice do not have this defect but die around two days later. Mfn1 deficient MEF cells have a more severe mitochondrial phenotype, with loss in potential and motility [78]. This would suggest that Mfn1 plays a more essential role in mediating fusion than Mfn2 or they may regulate fusion signaled from different pathways. While both Mfn1 and Mfn2 are important for mitochondrial fusion, a hydrolysis deficient form of Mfn2 was shown to stimulate mitochondrial fusion in cultured cells, and protected cells from apoptosis induced by staurosporine [79], which indicated additional regulatory functions for Mfn2. These suggest that apart from the fusion role, Mfn2 may also have additional functions that may impact the survival of cells.

The third GTPase required for mitochondrial fusion is localized to the intermembrane space and is called Opa1 [86-89]. Opa1 (Optical Atrophy 1) is imported into the mitochondrial inner membrane where it undergoes a regulated cleavage event to release a soluble shorter form of the protein into the intermembrane space. Mutations in Opa1 are causal for autosomal dominant optical atrophy, characterized by degeneration of the retinal ganglial cells in the eye resulting in blindness [90, 91]. There are 8 splice variants of Opa1 in humans, and the ratio of these isoforms and their cleavage products has complicated the progress to determine the precise function of Opa1. Mgm1p (yeast ortholog of Opa1) is cleaved upon insertion in the membrane by a member of the rhomboid family of inner membrane serine proteases called Rbd1p/Pcp1p/Mdm38p [92, 93]. The human orthologue of Rbd1p is called PARL for presenilin associated rhomboid like protein [94-96]. Currently it is unclear as to the efficiency by which PARL cleaves the human Opa1, since it has been shown that another matrix protease of the AAA
ATPase family called Paraplegin may also be responsible for Opa1 cleavage and the regulation of mitochondrial fusion [97]. Interestingly, similar to Opa1, deficiency in Paraplegin causes a type of neuronal degenerative disease called hereditary spastic paraplegia (HSP). HSP causes degeneration of corticalspinal axons, one of the longest in neuronal system [98]. Some forms of HSP, similar to mutations of Opa1 in humans, can also lead to retinopathy and optic neuropathies [99]. In addition to Paraplegin, the groups of de Strooper and Scorrano have shown that mice lacking PARL have reduced cleavage of Opa1, indicating a requirement for PARL activity in the maturation of Opa1 [96]. Comparing Opa1 processing and mitochondrial morphology between Opa1 and Paraplegin deficient mice [100] will show which protein is the major processor for Opa1. Parapelgin, however, has also been implicated in the proper ribosome assembly in mitochondria [101] and reduction of paraplegin can also induce complex 1 deficiency and sensitivity to oxidative stress [102]. The effect of paraplegin on Opa1 processing, therefore, may not be direct, and could be through other proteins such as PARL. Nonetheless, these studies show a requirement of proper Opa1 processing for its role in mitochondria, and the lost of proper Opa1 processing can specifically lead to neuronal degeneration.

\section{The role of mitochondrial fusion in cell death: Mfn1 and Mfn2 are not created equal in neuronal cell death}

In addition to the increase of mitochondrial fission, the fragmented mitochondria seen in apoptotic cells can also be due to an inhibition of fusion. Using photoactive mitochondrial labeling, it has been shown that mitochondrial fusion is blocked during Bax dependent apoptosis [29]. Recently, Bax has been shown to have a role in mitochondrial fusion by activating Mfn2 in healthy cells, therefore, it is possible that during cell death, mitochondrial fusion mediated by Bax is inhibited and thus mitochondrial fission is enhanced [103]. Interestingly, Drp1, Bax and Mfn2 have been shown to colocalize into discrete foci during apoptosis, suggesting that Mfn2 may be sequestered and inhibited during apoptosis [33]. Increased Mfn2 activity can inhibit Bax activation and cytochrome $c$ release $[69,79]$, whereas the lack of Mfn2 activity sensitize cells to apoptotic stimuli [69]. In neuronal system, Barsoum and colleagues demonstrated that overexpression of Mfn1 can protect against NO induced neuronal cell death. The overexpression of Mfn1, on the other hand, does not protect against excitotoxic induced cell death [30]. Thus, while Mfn1 and Mfn2 have common functions in terms of mitochondrial fusion, there are clearly some very important distinctions. In addition to the differences in nucleotide binding and hydrolysis as mentioned above, recently, compelling evidence has emerged supporting a signaling role 


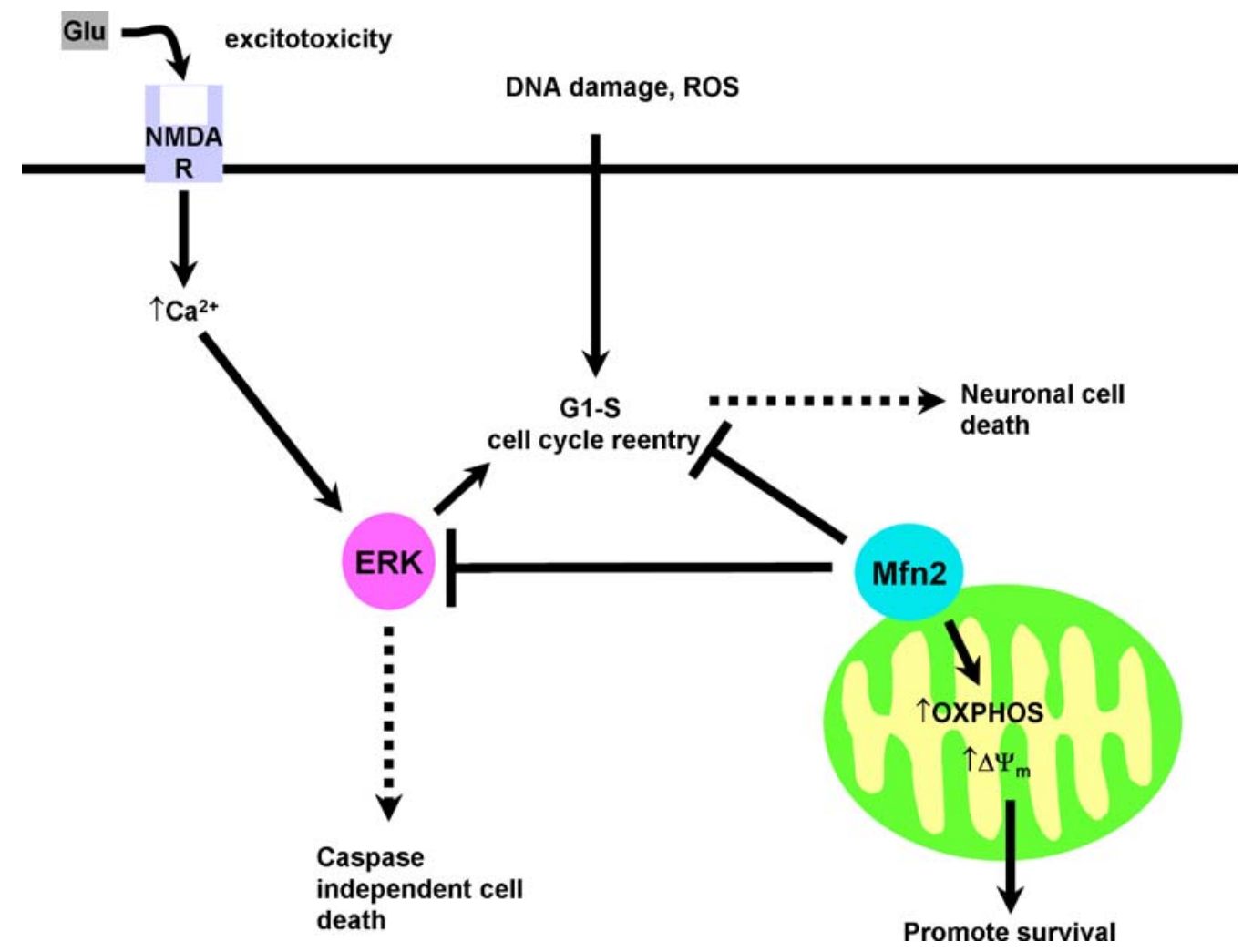

Fig. 2 Mfn2 may protect neurons via fusion independent mechanisms. Mfn2 can increase oxidative phosphorylation and mitochondrial membrane potential that can promote neuronal survival. Mfn2 can also

for Mfn2 that goes beyond mitochondrial fusion. Mfn2 (not Mfn1) is colocalized in punctae with Bax and Drp1 at sites of future fission [33]. This suggests that Mfn2 activity may affect mitochondrial recruitment of Bax or Drp1 during cell death. Mfn2 can also interact directly with Ced9 or BclxL in HEK293 cells suggesting a mechanism for crosstalk with antiapoptotic Bcl family proteins [71]. Mfn2 has been shown to modulate metabolism [104, 105] and cell cycle [106]. We therefore suggest that Mfn2, unlike Mfn1, may be able to provide additional protection against neuronal apoptosis through mitochondrial fusion independent mechanism, such as metabolic and cell cycle control (Fig. 2). In the following section we will discuss the mitochondrial fusion independent role of Mfn2 in metabolism, cell cycle control and apoptosis.

Studies in diabetic model systems have indicated that the reduction of Mfn2 leads to an inhibition of glucose oxidation and cell respiration, which is linked to obesity in both rat and human [104]. Mfn2 control of metabolism is not dependent on mitochondrial fusion, since expression of a truncated form of Mfn2 that is localized in cytoplasm can still increase mitochondrial respiration. This suggests that mitochondrial fusion and metabolic control by Mfn2 can be dissociated [105]. Mfn2 controls metabolic rate by increasing the expression of the oxidative phosphorylation proteins, however, the exact mechanism remains to be elucidated. Neurons require inhibit G1-2 cell cycle reentry and ERK activation during cell death. For details see text

a high energy supply not only for housekeeping, but also for maintaining the plasma membrane potential and firing of the action potential. Furthermore, neurons are highly dependant on oxidative phosphorylation for energy production [107]. Increased activation of Mfn2, therefore, may rescue neuronal cell death by maintaining functional oxidative phosphorylation and most importantly, mitochondrial membrane potential, both of which are disrupted in many models of neuronal cell death such as excitotoxicity and ROS (Fig. 2).

Mitochondrial fission/fusion activity has also been observed during cell cycle (for review see [41]). Mitochondrial undergo fragmentation when yeast cells enter into $M$ phase [108]. In the mammalian system, Mfn2 activation blocks G1 to $\mathrm{S}$ transition and cell cycle is arrested. This is achieved by inhibiting the MAPK signaling proteins Ras and ERK. Importantly, mutated Mfn2 that is not targeted to mitochondria does not impede the cell cycle control function, suggesting that Mfn2 control of cell cycle is fusion independent and Mfn2 function as a signaling GTPase [106]. There is compelling evidence that after induction of cell death, postmitotic neurons re-enter the cell cycle at G1-S and the deregulation of cell cycle proteins participate in cell death (for a detailed review see [109-112]). For example, DNA damage induced cell death triggers post-mitotic neurons to undergo $S$ phase reentry, and inhibition of the cell cycle machinery 
can protect neurons against DNA damage [113-116]. It is therefore possible that Mfn2 may inhibit neuronal cell death by blocking the G1 to $\mathrm{S}$ transition. The MAPK family protein ERK has also been suggested to act as a key factor in executing neuronal cell death (for review see $[117,118]$ ). In neurons, chronic activation of ERK at the nucleus induced by potassium withdrawal triggers non-classical caspase 3 independent cell death that is characterized by plasma membrane damage and nuclear condensation [119]. Activation of ERK has also been shown to be a key mediator in excitotoxicity [120] and ROS [121, 122]. By inhibiting ERK activation, Mfn2 may also inhibit neuronal cell death independent of mitochondrial fusion (Fig. 2) Taken together, these studies suggest that Mfn2 may function to inhibit neuronal cell death in a mitochondrial fusion independent manner, mechanistically distinct from other fusion proteins such as Opa1 and Mfn1.

In contrast to other cell types, neurons have a distinct shape: long processes called axons and dendrites extend away from the cell body where the nucleus resides. These neurites have an abundance of mitochondria for neuronal plasticity, generating ATP for synaptic vesicle release and maintaining plasma membrane potential. These mitochondria also serve as a buffer for $\mathrm{Ca}^{2+}$ ions. During excitotoxicity, the cell death insults signal through the NMDA receptor at the synaptic sites, which are far away from the cell body where the nucleus is. Pathological activation of NMDA receptors leads to an influx of ions such as $\mathrm{Na}^{+}$and $\mathrm{Ca}^{2+}$ which induces nNOS activity. It should be noted that NMDA receptors and nNOS are tightly coupled to each other through PSD95 at the synaptic sites [123, 124]. Because of this close proximity, $\mathrm{Ca}^{2+}$ can activate nNOS locally at the neurites far away from the cell body, and mitochondria must be strategically located close to these sites of entry to absorb the deleteriously high $\mathrm{Ca}^{2+}$ and prevent activation of nNOS. During cell death, mitochondria not only fragment, but often the fragmented mitochondria are collapsed around the nucleus. By enhancing fusion, either by inhibition of fission or activating fusion, the elongated mitochondria can reach throughout the length of the neurites (Fig. 3). These mitochondria can possibly absorb efficiently where the highest amount of $\mathrm{Ca}^{2+}$ is located and thus prevent activation of nNOS and subsequent ROS production at those sites (Fig. 4). A transient increase of mitochondrial fusion after acute neuronal insult could possibly protect cells from excitotoxicity. Both our group and Barsoum et al. have shown that fusion protects against ROS [30, 76], which can also lead to $\mathrm{Ca}^{2+}$ influx through TRMP7 channels [125]. In contrast to neuronal cell death, in HeLa cells mitochondrial fission can protect against death induced by the propagation of $\mathrm{Ca}^{2+}$ waves along the mitochondria [126]. Thus, mitochondrial dynamics may play distinct roles depending on the cell types and the type of injury incurred. The fission machinery may be required for general remodeling of the membranes during death whereas the fusion machinery may be protective by inhibiting apoptotic signaling cascades and allowing the spatial regulation of $\mathrm{Ca}^{2+}$ influx. Further studies are required to understand how fission/fusion machinery modulates cell death involving calcium in different mechanisms of cell death.

\section{Mitochondrial cristae structure and cell death: Demolition from within}

Apart from the dividing and fusing of the mitochondria, the mitochondrial ultrastructure also undergoes substantial changes during cell death, specifically, the cristae is remodeled when the cell is induced to die. In healthy mitochondria, the relatively tight cristae junction connects to the tubular and lamellar cristae. The morphological control of this cristae structure contributes to metabolic rate of mitochondria by generating a gradient of metabolites and substrates for oxidative phosphorylation and ATP synthesis. This tight cristae junction also provides a barrier for cytochrome $C$ such that approximately $80 \%$ of total cyt $c$ is in the cristae [127-129]. Scorrano and colleagues first showed that isolated mitochondria treated with the pro-apoptotic $\mathrm{BH} 3$ protein $\mathrm{tBid}$ induces cytochrome $c$ mobilization from the cristae to the intermembrane space, and thereby assist in BAX/BAK dependent release of cytochrome $c$ through MOMP. These mitochondrial changes induced by tBid are independent of BAX and BAK, but dependent on permeability transition pore components. EM studies show that the cristae junction is dilated in tBid treated mitochondria, thus allowing cytochrome $c$ to be mobilized through these dilated junctions to the intermembrane space [130]. The importance of cristae remodeling during cell death has also been shown by Germain and colleagues in a study of BIK mediated cell death through the ER pathway. In this model, cristae remodeling is dependent on calcium ions from ER and Drp1, suggesting a possible role of mitochcondrial fission protein in cristae remodeling during apoptosis [63].

Two recent studies by Scorrano and de Strooper group have shed light on the possible mechanism of cristae remodeling during cell death through the fusion protein Opa1 [96, 131]. Opa1 is processed from the long membrane anchored form of Opa1 (1-Opa1) to a soluble short form (s-Opa1). The long and short form of Opa 1 can form oligomers which form the narrow tubular cristae junction of cristae that contains the majority of cytochrome $c$. Most importantly, these Opa1 oligomers were lost in the mitochondria after tBid treatment and as a result the tight tubular cristae junction is dilated and cytochrome $c$ is mobilized, accelerating cell death progression. This is in agreement with previous Opa1 siRNA studies showing dilated cristae junction in mitochondria [132]. Indeed, Opa1 overexpression rescues cell death by maintaining 


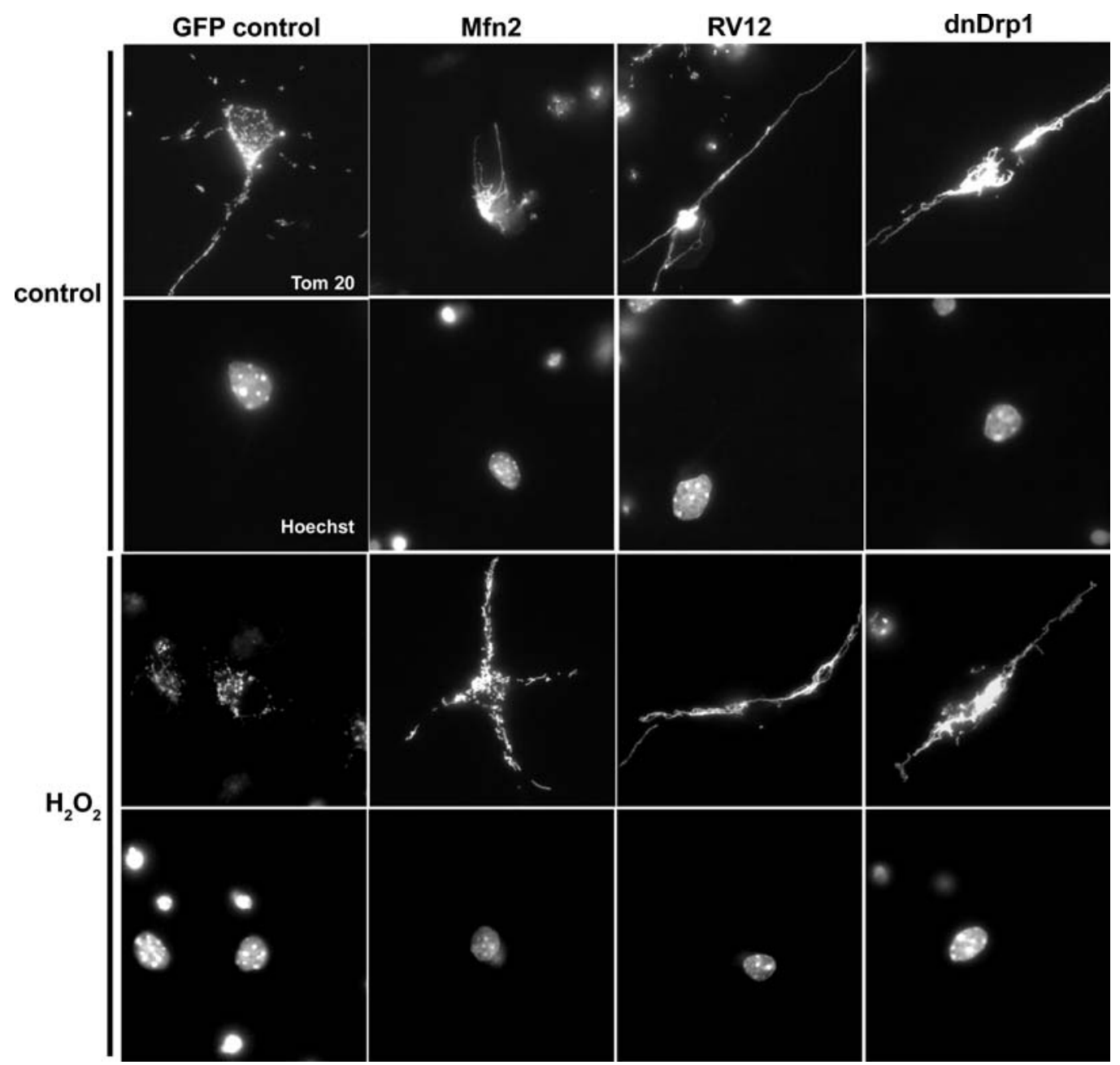

Fig. 3 Activation of the mitochondrial fusion protein Mfn2 or inhibition of the fission protein Drp1 can enhance mitochondrial fusion in cortical neurons, which can protect mitochondria from fragmentation during neuronal cell death induced by oxidative stress $\left(\mathrm{H}_{2} \mathrm{O}_{2}\right)$ and subsequently, inhibition of cell death [125]. Cortical neurons from E15.5

the tight cristae junction, which is independent of Mfn1/2. The role of Opa1 in inhibiting cytochrome $c$ mobilization by maintaining tight cristae junction is supported by PARL knockout studies. PARL ${ }^{-/-}$MEFs are more sensitive to cell death due to the lack of s-Opa1 which is essential for the formation of Opa1 oligomers. EM pictures of PARL ${ }^{-/-}$mitochondrial show faster cristae remodeling after tBid treatment, and cytochrome $c$ redistributed to the intermembrane space faster than wildtype, suggesting that correct processing of Opa1 and the subsequent Opa1 oligomer formation is essential for maintaining the tight cristae junction. These studies suggest that Opa1 oligomer formation by PARL controls the tight cristae junction that is essential to contain the majority of cytochrome $c$ in the cristae. During tBid induced apoptosis, the interaction between long and short form of Opa1 is disrupted, the cristae junction is dilated and cytochrome $c$ is mobilized and released into cytosol. As tBid is localized to mice were infected with the indicated construct via adenoviral vector at the time of plating. After $36 \mathrm{~h}$ neurons were treated with $\mathrm{H}_{2} \mathrm{O}_{2}$, fixed after $12 \mathrm{~h}$, and stained with Tom 20 to visualize mitochondria, and hoechst for nuclei

the outer membrane, the mechanism by which it disassembles Opa1 oligomers is unclear. It has been suggested that tBid interacts with cardiolipin at contact sites between inner and outer membrane and this interaction induces cristae morphological changes [133], possibly by tBid's lipid transfer activity [134] or tBid induced alteration of membrane curvature [135]. Opa1 has also been suggested to be released from mitochondria, which may lead to disruption of Opa1 oligomers [68]. Other proteins that regulate cristae morphology, such as Mitofilin which can form oligomers [136] and ATP synthase subunit e/g [137, 138] may also be involved in Opa1 control of cristae structure.

Recently, our study on the role of AIF in cell death provided another possible link on the relationship between the control of crisate morphology and cell death. Apart from the apoptotic role of AIF following its release and translocation to the nucleus [3, 139-142], AIF also has a physiological 


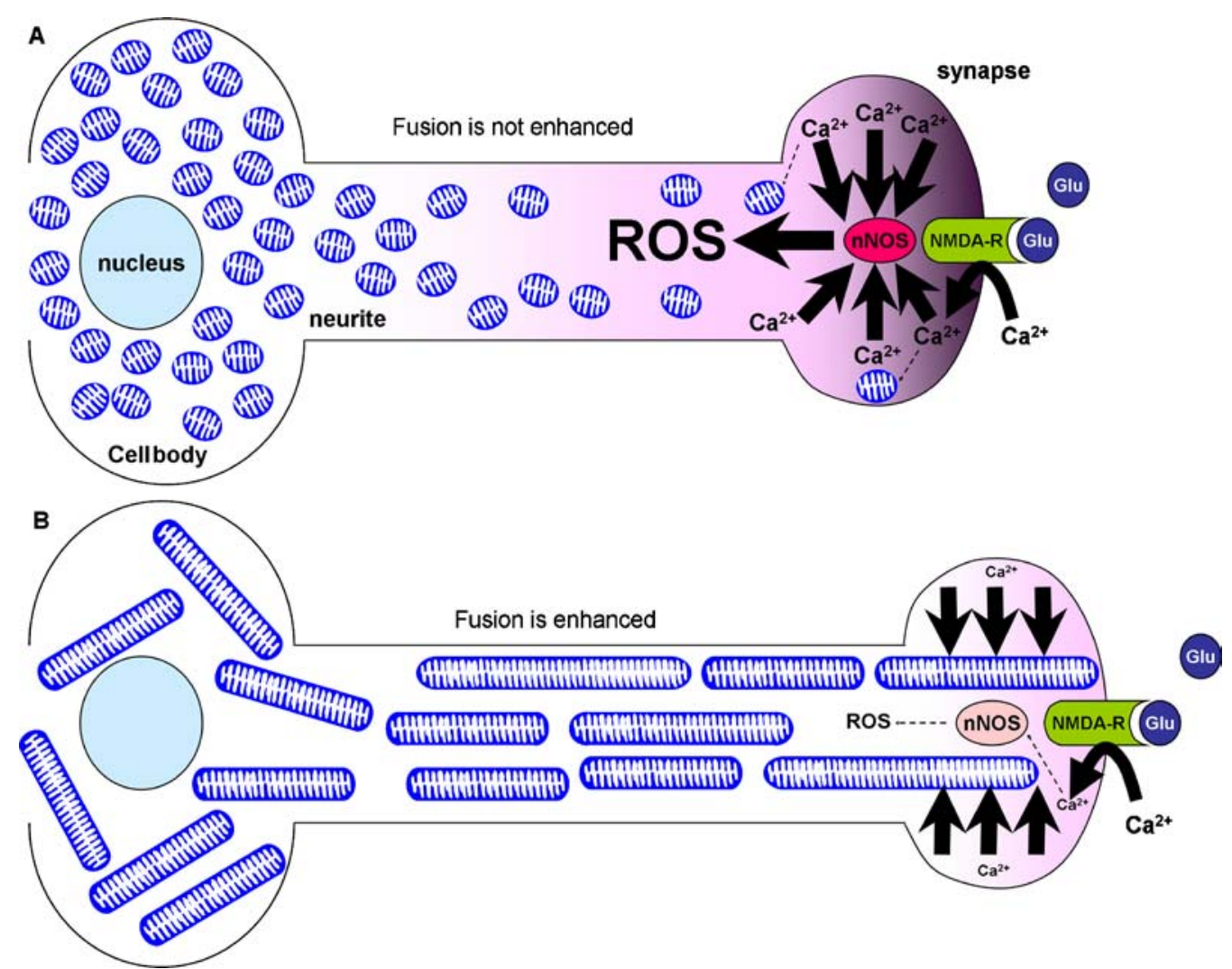

Fig. 4 During excitotoxicity, elongated mitochondria along the neurite and at the synapse can buffer $\mathrm{Ca}^{2+}$ more effectively than fragmented mitochondria. Hence increase in nNOS activity is moderate and less ROS is produced

role in mitochondria. AIF deficient cells emphasize the importance of AIF in maintaining mitochondrial function since these cells exhibit a loss of complex 1 stability and activity, and disruption of oxidative phosphorylation [143, 144]. In order to separate the function of the mitochondrial AIF from the nuclear functions of AIF during cell death, we constructed anchored AIF mutants which are anchored in the inner membrane of mitochondria and cannot be released during apoptosis. Since whole embryo AIF knockouts are embryonic lethal, we generated conditional forebrain deficient AIF mice to study the role of AIF in neurons. We first observed that $\mathrm{AIF}^{-/-}$neurons exhibited fragmented mitochondria with dilated mitochondrial cristae and defective mitochondrial respiration, which is similar to Opa1 knockdown cells [132, 145]. EM studies of neurons expressing the unreleasable form of AIF showed elongated mitochondria and tighter cristae tubules. Importantly, expression of the unreleasable form of AIF in neurons could delay cell death by slowing down the release of cytochrome $c$ from the mitochondria. From these results we suggest that AIF may control the cristae structure, possibly through interactions with other proteins, to retain cytochrome $C$ within cristae. This cristae structure is disrupted during cell death when AIF is released. Although the maintenance of AIF within the mitochondria delayed MOMP in the null background, the apoptotic role of
AIF in the nucleus is, however, still a very important aspect of AIF function during cell death. In neurons that have the endogenous pool of AIF that can be translocated to the nucleus, the presence of mitochondrial anchored AIF does not delay cytochrome $C$ release and inhibit cell death at longer time points, indicating that AIF is still an important factor in neuronal apoptosis following its release and translocation to the nucleus [142]. In conclusion, these results show that apart from Bax/Bak control of the release of apoptotic factors, mitochondrial itself can also control the release of cytochrome $c$ by remodeling the cristae utilizing its own intermembrane space proteins such as Opa1 and AIF.

\section{Conclusion}

Recent studies have demonstrated that the mitochondrial fission and fusion machinery may play an important role in cell death. Mitochondrial fission is an early event during neuronal cell death, and by inhibiting the fission machinery, cell death is inhibited after various neurotoxic insults. It has also been shown that in non-neuronal cells the induction of fission is not always sufficient to induce cell death. Other factors such as the proapoptotic $\mathrm{Bcl}-2$ family members must be activated to execute cell death, since fission occurs in 
physiological situations such as cell division without going into apoptosis. Other factors clearly remain to be discovered for the activation of mitochondrial fission machinery to drive cell death after different types of neuronal injuries. Fusion proteins may protect against neuronal cell death not only by their ability to inhibit mitochondrial fission, but also by their fission/fusion independent roles as signaling molecules for other pathways. By studying the interaction among mitochondrial fission/fusion machinery, Bcl-2 family proteins and metabolic proteins, we will gain insights as to how different types of neuronal cell death is regulated through diverse pathways that converge on mitochondria, which act as interpreters of multiple signals that ultimately determine the fate of the neuron after cell death induction.

Acknowledgments This work was supported by grants from CIHR and Heart and Stroke Foundation of Canada to RSS, and CIHR to HMM. ECC was supported by a CIHR studentship.

\section{References}

1. Danial NN, Korsmeyer SJ (2004) Cell death: critical control points. Cell 116(2):205-219

2. Green DR, Kroemer G (2004) The pathophysiology of mitochondrial cell death. Science 305(5684):626-629

3. Cregan SP, Dawson VL, Slack RS (2004) Role of AIF in caspasedependent and caspase-independent cell death. Oncogene 23(16):2785-2796

4. Aarts MM, Tymianski M (2004) Molecular mechanisms underlying specificity of excitotoxic signaling in neurons. Curr Mol Med 4(2):137-147

5. Yuan J, Lipinski M, Degterev A (2003) Diversity in the mechanisms of neuronal cell death. Neuron 40(2):401-413

6. Velier JJ et al (1999) Caspase-8, caspase-3 are expressed by different populations of cortical neurons undergoing delayed cell death after focal stroke in the rat. J Neurosci 19(14):5932-5941

7. Benchoua A et al (2001) Specific caspase pathways are activated in the two stages of cerebral infarction. J Neurosci 21(18):7127-7134

8. Lindsten T, Zong WX, Thompson CB (2005) Defining the role of the Bcl-2 family of proteins in the nervous system. Neuroscientist 11(1):10-15

9. Cheung EC et al (2006) Dissociating the dual roles of apoptosisinducing factor in maintaining mitochondrial structure and apoptosis. Embo J 25(17):4061-4073

10. Dargusch $\mathrm{R}$ et al (2001) The role of Bax in glutamate-induced nerve cell death. J Neurochem 76(1):295-301

11. Dirnagl U, Iadecola C, Moskowitz MA (1999) Pathobiology of ischaemic stroke: an integrated view. Trends Neurosci 22(9):391-397

12. Rothman SM, Olney JW (1986) Glutamate and the pathophysiology of hypoxic-ischemic brain damage. Ann Neurol 19(2):105-111

13. Schwarcz R, Whetsell Jr WO (1982) Post-mortem high affinity glutamate uptake in human brain. Neuroscience 7(7):1771-1778

14. Choi DW (1988) Glutamate neurotoxicity and diseases of the nervous system. Neuron 1(8):623-634

15. Collins RC, Dobkin BH, Choi DW (1989) Selective vulnerability of the brain: new insights into the pathophysiology of stroke. Ann Intern Med 110(12):992-1000
16. Meyer FB (1989) Calcium, neuronal hyperexcitability and ischemic injury. Brain Res Brain Res Rev 14(3):227-243

17. Siesjo BK (1988) Mechanisms of ischemic brain damage. Crit Care Med 16(10):954-963

18. Siesjo BK (1988) Historical overview. Calcium, ischemia, and death of brain cells. Ann N Y Acad Sci 522:638-661

19. Arundine M, Tymianski M (2003) Molecular mechanisms of calcium-dependent neurodegeneration in excitotoxicity. Cell Calcium 34(4-5):325-337

20. Castilho RF, Kowaltowski AJ, Vercesi AE (1998) 3,5,3'triiodothyronine induces mitochondrial permeability transition mediated by reactive oxygen species and membrane protein thiol oxidation. Arch Biochem Biophys 354(1):151-157

21. Dawson VL et al (1991) Nitric oxide mediates glutamate neurotoxicity in primary cortical cultures. Proc Natl Acad Sci USA 88(14):6368-6371

22. Stout AK et al (1998) Glutamate-induced neuron death requires mitochondrial calcium uptake. Nat Neurosci 1(5):366-373

23. Beckman JS (1991) The double-edged role of nitric oxide in brain function and superoxide-mediated injury. J Dev Physiol 15(1):53-59

24. Beckman JS, Crow JP (1993) Pathological implications of nitric oxide, superoxide and peroxynitrite formation. Biochem Soc Trans 21(2):330-334

25. Sattler R, Tymianski M (2000) Molecular mechanisms of calcium-dependent excitotoxicity. J Mol Med 78(1):3-13

26. Sattler R, Tymianski M (2001) Molecular mechanisms of glutamate receptor-mediated excitotoxic neuronal cell death. Mol Neurobiol 24(1-3):107-129

27. Zamzami N, Kroemer G (2001) The mitochondrion in apoptosis: how Pandora's box opens. Nat Rev Mol Cell Biol 2(1):67-71

28. Frank $S$ et al (2001) The role of dynamin-related protein 1, a mediator of mitochondrial fission, in apoptosis. Dev Cell 1(4):515-525

29. Karbowski M et al (2004) Quantitation of mitochondrial dynamics by photolabeling of individual organelles shows that mitochondrial fusion is blocked during the Bax activation phase of apoptosis. J Cell Biol 164(4):493-499

30. Barsoum MJ et al (2006) Nitric oxide-induced mitochondrial fission is regulated by dynamin-related GTPases in neurons. Embo J 25(16):3900-3911

31. Arnoult D et al (2005) Bax/Bak-dependent release of DDP/TIMM8a promotes Drp1-mediated mitochondrial fission and mitoptosis during programmed cell death. Curr Biol 15(23):2112-2118

32. Chen H, Chan DC (2005) Emerging functions of mammalian mitochondrial fusion and fission. Hum Mol Genet 14(Spec 2):R283-R289

33. Karbowski M et al (2002) Spatial and temporal association of Bax with mitochondrial fission sites, Drp1, and Mfn2 during apoptosis. J Cell Biol 159(6):931-938

34. Osteryoung KW, Nunnari J (2003) The division of endosymbiotic organelles. Science 302(5651):1698-1704

35. Chan DC (2006) Mitochondria: dynamic organelles in disease, aging, and development. Cell 125(7):1241-1252

36. van der Bliek AM (2000) A mitochondrial division apparatus takes shape. J Cell Biol 151(2):F1-F4

37. Meeusen SL, Nunnari J (2005) How mitochondria fuse. Curr Opin Cell Biol 17(4):389-394

38. Jensen RE (2005) Control of mitochondrial shape. Curr Opin Cell Biol 17(4):384-388

39. Westermann B (2002) Merging mitochondria matters: cellular role and molecular machinery of mitochondrial fusion. EMBO Rep 3(6):527-531

40. Mozdy AD, Shaw JM (2003) A fuzzy mitochondrial fusion apparatus comes into focus. Nat Rev Mol Cell Biol 4(6):468-478 
41. McBride HM, Neuspiel M, Wasiak S (2006) Mitochondria: more than just a powerhouse. Curr Biol 16(14):R551-R560

42. Jagasia $R$ et al (2005) DRP-1-mediated mitochondrial fragmentation during EGL-1-induced cell death in C. elegans. Nature 433(7027):754-760

43. Youle RJ, Karbowski M (2005) Mitochondrial fission in apoptosis. Nat Rev Mol Cell Biol 6(8):657-663

44. Chang DT, Reynolds IJ (2006) Differences in mitochondrial movement and morphology in young and mature primary cortical neurons in culture. Neuroscience 141(2):727-736

45. Li, Z et al (2004) The importance of dendritic mitochondria in the morphogenesis and plasticity of spines and synapses. Cell 119(6):873-887

46. Heath-Engel HM, Shore GC (2006) Mitochondrial membrane dynamics, cristae remodelling and apoptosis. Biochim Biophys Acta 1763(5-6):549-560

47. Perfettini JL, Roumier T, Kroemer G (2005) Mitochondrial fusion and fission in the control of apoptosis. Trends Cell Biol 15(4):179-183

48. Mukamel Z, Kimchi A (2004) Death-associated protein 3 localizes to the mitochondria and is involved in the process of mitochondrial fragmentation during cell death. J Biol Chem 279(35):36732-36738

49. Niemann A et al (2005) Ganglioside-induced differentiation associated protein 1 is a regulator of the mitochondrial network: new implications for Charcot-Marie-Tooth disease. J Cell Biol 170(7):1067-1078

50. Tondera D et al (2005) The mitochondrial protein MTP18 contributes to mitochondrial fission in mammalian cells. J Cell Sci 118(Pt 14):3049-3059

51. James DI et al (2003) hFis1, a novel component of the mammalian mitochondrial fission machinery. J Biol Chem 278(38):36373-36379

52. Yoon $Y$ et al (2003) The mitochondrial protein hFis1 regulates mitochondrial fission in mammalian cells through an interaction with the dynamin-like protein DLP1. Mol Cell Biol 23(15):5409-5420

53. Ingerman E et al (2005) Dnm1 forms spirals that are structurally tailored to fit mitochondria. J Cell Biol 170(7):1021-1027

54. Okamoto K, Shaw JM (2005) Mitochondrial morphology and dynamics in yeast and multicellular eukaryotes. Annu Rev Genet 39:503-536

55. Karbowski M, Jeong SY, Youle RJ (2004) Endophilin B1 is required for the maintenance of mitochondrial morphology. $\mathrm{J}$ Cell Biol 166(7):1027-1039

56. Yoon Y (2004) Sharpening the scissors: mitochondrial fission with aid. Cell Biochem Biophys 41(2):193-206

57. Lee YJ et al (2004) Roles of the mammalian mitochondrial fission and fusion mediators Fis1, Drp1, and Opa1 in apoptosis. Mol Biol Cell 15(11):5001-5011

58. Harder Z, Zunino R, McBride H (2004) Sumol conjugates mitochondrial substrates and participates in mitochondrial fission. Curr Biol 14(4):340-345

59. Martinou I et al (1999) The release of cytochrome $c$ from mitochondria during apoptosis of NGF-deprived sympathetic neurons is a reversible event. J Cell Biol 144(5):883-889

60. Sheridan JW, Bishop CJ, Simmons RJ (1981) Biophysical and morphological correlates of kinetic change and death in a starved human melanoma cell line. J Cell Sci 49:119-137

61. Zhuang J, Dinsdale D, Cohen GM (1998) Apoptosis, in human monocytic THP. 1 cells, results in the release of cytochrome $c$ from mitochondria prior to their ultracondensation, formation of outer membrane discontinuities and reduction in inner membrane potential. Cell Death Differ 5(11):953-962

62. Martinou JC, Youle RJ (2006) Which came first, the cytochrome $c$ release or the mitochondrial fission? Cell Death Differ
13(8):1291-1295

63. Germain M et al (2005) Endoplasmic reticulum BIK initiates DRP1-regulated remodelling of mitochondrial cristae during apoptosis. Embo J 24(8):1546-1556

64. Breckenridge DG et al (2003) Caspase cleavage product of BAP31 induces mitochondrial fission through endoplasmic reticulum calcium signals, enhancing cytochrome $c$ release to the cytosol. J Cell Biol 160(7):1115-1127

65. Pierrat B et al (2001) SH3GLB, a new endophilin-related protein family featuring an SH3 domain. Genomics 71(2):222-334

66. Wei MC et al (2001) Proapoptotic BAX and BAK: a requisite gateway to mitochondrial dysfunction and death. Science 292(5517):727-730

67. Esseiva AC et al (2004) Temporal dissection of Bax-induced events leading to fission of the single mitochondrion in Trypanosoma brucei. EMBO Rep 5(3):268-273

68. Arnoult D et al (2005) Release of OPA1 during apoptosis participates in the rapid and complete release of cytochrome $c$ and subsequent mitochondrial fragmentation. J Biol Chem 280(42):35742-35750

69. Sugioka R, Shimizu S, Tsujimoto Y (2004) Fzo1, a protein involved in mitochondrial fusion, inhibits apoptosis. J Biol Chem 279(50):52726-52734

70. Kuwana T et al (2002) Bid, Bax, and lipids cooperate to form supramolecular openings in the outer mitochondrial membrane. Cell 111(3):331-342

71. Delivani P et al (2006) Role for CED-9 and Egl-1 as regulators of mitochondrial fission and fusion dynamics. Mol Cell 21(6):761-773

72. Parone PA et al (2006) Inhibiting the mitochondrial fission machinery does not prevent Bax/Bak-dependent apoptosis. Mol Cell Biol 26(20):7397-7408

73. Alirol E et al (2006) The Mitochondrial Fission Protein hFis1 Requires the Endoplasmic Reticulum Gateway to Induce Apoptosis. Mol Biol Cell 17(11):4593-4605

74. Yethon JA et al (2003) Interaction with a membrane surface triggers a reversible conformational change in Bax normally associated with induction of apoptosis. J Biol Chem 278(49):48935-48941

75. Basanez G et al (2002) Bax-type apoptotic proteins porate pure lipid bilayers through a mechanism sensitive to intrinsic monolayer curvature. J Biol Chem 277(51):49360-49365

76. Jahani-Asl A, Cheung ECC, Neuspiel M, MacLaurin JG, Fortin A, Park DS, McBride H, Slack RS (2006) Mitofusin 2 Protects Cerebellar Granule Neurons Against Acute Injury. submitted

77. Chen H, Chomyn A, Chan DC (2005) Disruption of fusion results in mitochondrial heterogeneity and dysfunction. J Biol Chem 280(28):26185-26192

78. Chen H et al (2003) Mitofusins Mfn1 and Mfn2 coordinately regulate mitochondrial fusion and are essential for embryonic development. J Cell Biol 160(2):189-200

79. Neuspiel $\mathrm{M}$ et al (2005) Activated mitofusin 2 signals mitochondrial fusion, interferes with Bax activation, and reduces susceptibility to radical induced depolarization. J Biol Chem 280(26):25060-25070

80. Eura Y et al (2003) Two mitofusin proteins, mammalian homologues of FZO, with distinct functions are both required for mitochondrial fusion. J Biochem (Tokyo) 134(3):333344

81. Rojo M et al (2002) Membrane topology and mitochondrial targeting of mitofusins, ubiquitous mammalian homologs of the transmembrane GTPase Fzo. J Cell Sci 115(Pt 8):16631674

82. Santel A et al (2003) Mitofusin-1 protein is a generally expressed mediator of mitochondrial fusion in mammalian cells. J Cell Sci 116(Pt 13):2763-2774 
83. Zuchner S et al (2004) Mutations in the mitochondrial GTPase mitofusin 2 cause Charcot-Marie-Tooth neuropathy type 2A. Nat Genet 36(5):449-451

84. Cipolat $\mathrm{S}$ et al (2004) OPA1 requires mitofusin 1 to promote mitochondrial fusion. Proc Natl Acad Sci USA 101(45):15927-15932

85. Ishihara N, Eura Y, Mihara K (2004) Mitofusin 1 and 2 play distinct roles in mitochondrial fusion reactions via GTPase activity. J Cell Sci 117(Pt 26):6535-6546

86. Olichon A et al (2002) The human dynamin-related protein OPA1 is anchored to the mitochondrial inner membrane facing the inter-membrane space. FEBS Lett 523(1-3):171-176

87. Alexander C et al (2000) OPA1, encoding a dynamin-related GTPase, is mutated in autosomal dominant optic atrophy linked to chromosome 3q28. Nat Genet 26(2):211-215

88. Delettre $\mathrm{C}$ et al (2000) Nuclear gene OPA1, encoding a mitochondrial dynamin-related protein, is mutated in dominant optic atrophy. Nat Genet 26(2):207-210

89. Delettre $\mathrm{C}$ et al (2001) Mutation spectrum and splicing variants in the OPA1 gene. Hum Genet 109(6):584-591

90. Delettre $\mathrm{C}$ et al (2000) Nuclear gene OPA1, encoding a mitochondrial dynamin-related protein, is mutated in dominant optic atrophy. Nat Genet 26(2):207-210

91. Delettre C et al (2001) Mutation spectrum and splicing variants in the OPA1 gene. Hum Genet 109(6):584-591

92. Herlan M et al (2003) Processing of Mgm1 by the rhomboid-type protease Pcp1 is required for maintenance of mitochondrial morphology and of mitochondrial DNA. J Biol Chem 278(30):27781-27788

93. Sesaki H et al (2003) Cells lacking Pcp1p/Ugo2p, a rhomboidlike protease required for Mgm1p processing, lose mtDNA and mitochondrial structure in a Dnm1p-dependent manner, but remain competent for mitochondrial fusion. Biochem Biophys Res Commun 308(2):276-283

94. Sik A et al (2004) Self-regulated cleavage of the mitochondrial intramembrane-cleaving protease PARL yields Pbeta, a nuclear-targeted peptide. J Biol Chem 279(15):15323-15329

95. McQuibban GA, Saurya S, Freeman M (2003) Mitochondrial membrane remodelling regulated by a conserved rhomboid protease. Nature 423(6939):537-541

96. Cipolat $\mathrm{S}$ et al (2006) Mitochondrial rhomboid PARL regulates cytochrome $c$ release during apoptosis via OPA1-dependent cristae remodeling. Cell 126(1):163-175

97. Ishihara $\mathrm{N}$ et al (2006) Regulation of mitochondrial morphology through proteolytic cleavage of OPA1. Embo J 25(13):29662977

98. Casari G et al (1998) Spastic paraplegia and OXPHOS impairment caused by mutations in paraplegin, a nuclear-encoded mitochondrial metalloprotease. Cell 93(6):973-983

99. Harding AE (1983) Classification of the hereditary ataxias and paraplegias. Lancet 1(8334):1151-1155

100. Ferreirinha $\mathrm{F}$ et al (2004) Axonal degeneration in paraplegindeficient mice is associated with abnormal mitochondria and impairment of axonal transport. J Clin Invest 113(2):231-242

101. Nolden $M$ et al (2005) The m-AAA protease defective in hereditary spastic paraplegia controls ribosome assembly in mitochondria. Cell 123(2):277-289

102. Atorino L et al (2003) Loss of m-AAA protease in mitochondria causes complex I deficiency and increased sensitivity to oxidative stress in hereditary spastic paraplegia. J Cell Biol 163(4):777-787

103. Karbowski M et al (2006) Role of Bax and Bak in mitochondrial morphogenesis. Nature 443(7112):658-662

104. Bach D et al (2003) Mitofusin-2 determines mitochondrial network architecture and mitochondrial metabolism. A novel regulatory mechanism altered in obesity. J Biol Chem 278(19):17190-17197
105. Pich $\mathrm{S}$ et al (2005) The Charcot-Marie-Tooth type 2A gene product, Mfn2, up-regulates fuel oxidation through expression of OXPHOS system. Hum Mol Genet 14(11):1405-1415

106. Chen KH et al (2004) Dysregulation of HSG triggers vascular proliferative disorders. Nat Cell Biol 6(9):872-883

107. Gjedde A, Marrett S, Vafaee M (2002) Oxidative and nonoxidative metabolism of excited neurons and astrocytes. J Cereb Blood Flow Metab 22(1):1-14

108. Tanaka K, Kanbe T, Kuroiwa T (1985) Three-dimensional behaviour of mitochondria during cell division and germ tube formation in the dimorphic yeast Candida albicans. J Cell Sci 73:207-220

109. Liu DX, Greene LA (2001) Neuronal apoptosis at the G1/S cell cycle checkpoint. Cell Tissue Res 305(2):217-228

110. Herrup K et al (2004) Divide and die: cell cycle events as triggers of nerve cell death. J Neurosci 24(42):9232-9239

111. Nguyen MD, Mushynski WE, Julien JP (2002) Cycling at the interface between neurodevelopment and neurodegeneration. Cell Death Differ 9(12):1294-1306

112. Park DS et al (2000) Cell cycle regulators in neuronal death evoked by excitotoxic stress: implications for neurodegeneration and its treatment. Neurobiol Aging 21(6):771-781

113. Park DS et al (2000) Involvement of retinoblastoma family members and E2F/DP complexes in the death of neurons evoked by DNA damage. J Neurosci 20(9):3104-3114

114. Park DS et al (1997) G1/S cell cycle blockers and inhibitors of cyclin-dependent kinases suppress camptothecin-induced neuronal apoptosis. J Neurosci 17(4):1256-1270

115. Kruman II et al (2004) Cell cycle activation linked to neuronal cell death initiated by DNA damage. Neuron 41(4):549-561

116. Park DS et al (1998) Cyclin-dependent kinases participate in death of neurons evoked by DNA-damaging agents. J Cell Biol 143(2):457-467

117. Cheung EC, Slack RS (2004) Emerging role for ERK as a key regulator of neuronal apoptosis. Sci STKE 251:PE45

118. Subramaniam S, Unsicker K (2006) Extracellular signalregulated kinase as an inducer of non-apoptotic neuronal death. Neuroscience 138(4):1055-1065

119. Subramaniam $\mathrm{S}$ et al (2004) ERK activation promotes neuronal degeneration predominantly through plasma membrane damage and independently of caspase-3. J Cell Biol 165(3):357-369

120. Hughes JP et al (2003) Mitogen and stress response kinase-1 (MSK1) mediates excitotoxic induced death of hippocampal neurones. J Neurochem 86(1):25-32

121. Levinthal DJ, Defranco DB (2005) Reversible oxidation of ERK-directed protein phosphatases drives oxidative toxicity in neurons. J Biol Chem 280(7):5875-5883

122. Chu CT et al (2004) Oxidative neuronal injury. The dark side of ERK1/2. Eur J Biochem 271(11):2060-2066

123. Aarts $M$ et al (2002) Treatment of ischemic brain damage by perturbing NMDA receptor-PSD-95 protein interactions. Science 298(5594):846-850

124. Sattler R et al (1999) Specific coupling of NMDA receptor activation to nitric oxide neurotoxicity by PSD-95 protein. Science 284(5421): 1845-1848

125. Aarts $\mathrm{M}$ et al (2003) A key role for TRPM7 channels in anoxic neuronal death. Cell 115(7):863-877

126. Szabadkai $G$ et al (2004) Drp-1-dependent division of the mitochondrial network blocks intraorganellar $\mathrm{Ca}^{2+}$ waves and protects against $\mathrm{Ca}^{2+}$-mediated apoptosis. Mol Cell) This work is highly controversial and other labs have had opposite results (Ref. 1 and 2) 16(1):59-68

127. Mannella CA (2006) The relevance of mitochondrial membrane topology to mitochondrial function. Biochim Biophys Acta 1762(2):140-147

128. Mannella CA (2006) Structure and dynamics of the mito- 
chondrial inner membrane cristae. Biochim Biophys Acta 1763(5-6):542-548

129. Frey TG, Mannella CA (2000) The internal structure of mitochondria. Trends Biochem Sci 25(7):319-324

130. Scorrano L et al (2002) A distinct pathway remodels mitochondrial cristae and mobilizes cytochrome $c$ during apoptosis. Dev Cell 2(1):55-67

131. Frezza $\mathrm{C}$ et al (2006) OPA1 controls apoptotic cristae remodeling independently from mitochondrial fusion. Cell 126(1):177189

132. Griparic L et al (2004) Loss of the intermembrane space protein Mgm1/OPA1 induces swelling and localized constrictions along the lengths of mitochondria. J Biol Chem 279(18):1879218798

133. Kim TH et al (2004) Bid-cardiolipin interaction at mitochondrial contact site contributes to mitochondrial cristae reorganization and cytochrome $C$ release. Mol Biol Cell 15(7):30613072

134. Degli Esposti M, Dive C (2003) Mitochondrial membrane permeabilisation by Bax/Bak. Biochem Biophys Res Commun 304(3):455-461

135. Epand RF et al (2002) The apoptotic protein tBid promotes leakage by altering membrane curvature. J Biol Chem 277(36):32632-32639

136. John GB et al (2005) The mitochondrial inner membrane protein mitofilin controls cristae morphology. Mol Biol Cell 16(3):1543-1554
137. Arselin G et al (2004) The modulation in subunits e and $g$ amounts of yeast ATP synthase modifies mitochondrial cristae morphology. J Biol Chem 279(39):40392-40399

138. Paumard P et al (2002) The ATP synthase is involved in generating mitochondrial cristae morphology. Embo J 21(3):221-230

139. Cregan SP et al (2002) Apoptosis-inducing factor is involved in the regulation of caspase-independent neuronal cell death. J Cell Biol 158(3):507-517

140. Yu SW et al (2002) Mediation of poly(ADP-ribose) polymerase1-dependent cell death by apoptosis-inducing factor. Science 297(5579):259-263

141. Hong SJ, Dawson TM, Dawson VL (2004) Nuclear and mitochondrial conversations in cell death: PARP-1 and AIF signaling. Trends Pharmacol Sci 25(5):259-264

142. Cheung EC et al (2006) Dissociating the dual roles of apoptosisinducing factor in maintaining mitochondrial structure and apoptosis. Embo J 25(17):4061-4073

143. Joza $\mathrm{N}$ et al (2005) Muscle-specific loss of apoptosisinducing factor leads to mitochondrial dysfunction, skeletal muscle atrophy, and dilated cardiomyopathy. Mol Cell Biol 25(23): 10261-10272

144. Vahsen $\mathrm{N}$ et al (2004) AIF deficiency compromises oxidative phosphorylation. Embo J 23(23):4679-4689

145. Olichon A et al (2002) The human dynamin-related protein OPA1 is anchored to the mitochondrial inner membrane facing the inter-membrane space. FEBS Lett 523(1-3):171176 